\title{
Near-wellbore modeling of a horizontal well with Computational Fluid Dynamics
}

\section{Szanyi, Márton L.; Hemmingsen, Casper Schytte; Yan, Wei; Walther, Jens Honore; Glimberg, Stefan L.}

Published in:

Journal of Petroleum Science and Engineering

Link to article, DOI:

10.1016/j.petrol.2017.10.011

Publication date:

2018

Document Version

Peer reviewed version

Link back to DTU Orbit

Citation (APA):

Szanyi, M. L., Hemmingsen, C. S., Yan, W., Walther, J. H., \& Glimberg, S. L. (2018). Near-wellbore modeling of a horizontal well with Computational Fluid Dynamics. Journal of Petroleum Science and Engineering, 160, 119128. https://doi.org/10.1016/j.petrol.2017.10.011

\section{General rights}

Copyright and moral rights for the publications made accessible in the public portal are retained by the authors and/or other copyright owners and it is a condition of accessing publications that users recognise and abide by the legal requirements associated with these rights.

- Users may download and print one copy of any publication from the public portal for the purpose of private study or research.

- You may not further distribute the material or use it for any profit-making activity or commercial gain

- You may freely distribute the URL identifying the publication in the public portal 
Blue fonts: Manuscript was modified based on the Reviewer's comments.

Title: Near-wellbore modeling of a horizontal well with Computational Fluid Dynamics

Authors: Márton L. Szanyi ${ }^{1}$, Casper S. Hemmingsen ${ }^{1}$, Wei Yan ${ }^{1}$, Jens H. Walther ${ }^{1,3}$, Stefan L. Glimberg ${ }^{2}$

Abstract: The oil production by horizontal wells is a complex phenomenon that involves flow through the porous reservoir, completion interface and the well itself. Conventional reservoir simulators can hardly resolve the flow through the completion into the wellbore. On the contrary, Computational Fluid Dynamics (CFD) is capable of modeling the complex interaction between the creeping reservoir flow and turbulent well flow for single phases, while capturing both the completion geometry and formation damage. A series of single phase steady-state simulations are undertaken, using such fully coupled three dimensional numerical models, to predict the inflow to the well. The present study considers the applicability of CFD for near-wellbore modeling through benchmark cases with available analytical solutions. Moreover, single phase steady-state numerical investigations are performed on a specific perforated horizontal well producing from the Siri field, offshore Denmark. The performance of the well is investigated with an emphasis on the inflow profile and the productivity index for different formation damage scenarios. A considerable redistribution of the inflow profile were found when the filtrate invasion extended beyond the tip of the perforations.

Keywords: horizontal well productivity, near-wellbore model, inflow performance, reduced order model, numerical model, computational fluid dynamics.

Highlights: Computational Fluid Dynamics (CFD) simulation for modeling well inflow is introduced. Infinite conductivity horizontal wells can be modeled with CFD. The completion geometry can be incorporated into the numerical model. The inflow performance of the horizontal well in Siri field is assessed with CFD. Flow redistribution is observed for formation damage cases.

\section{Introduction}

Production by horizontal wells is a widely used technology of the upstream oil \& gas industry since several decades. The enhanced well-reservoir contact increases the area swept by the well, leading to a rise of inflow performance, thereby horizontal wells perform significantly better in thin reservoirs than vertical wells and reduce problems related with water or gas coning. However, the estimation of the horizontal well productivity is more challenging than the corresponding estimation for vertical wells. The partial penetration of the well into the reservoir, and the finite conductivity of the long wellbore results in a complex well-reservoir interaction that can hardly be captured by conventional analytical methods. Therefore, analytical formulas are only available for simplified problems where the flow in the wellbore is neglected and - in most cases - uniform well pressure assumed(Joshi, 1988), (Giger, et al., 1984), (Renard \& Dupuy, 1991). More sophisticated semi-analytical models were developed to overcome the uniform well pressure assumption, by including the pressure loss in the wellbore (Ozkan, et al., 1999). The inflow predicted by such finite conductivity well models reflect the field observations of increased production rate at the heel, where coning is more likely to occur. Nonetheless, when formation heterogeneity or complex well completion is present analytical or semi-analytical solutions are impossible to obtain, numerical methods must be used.

Standard large scale reservoir simulators are extensively used in the industry. However, they often lack the accuracy to resolve the well and the nearwellbore area since the applied Cartesian grid size (50 - $100 \mathrm{~m}$ ) is two orders of magnitude larger than the diameter of the well. Therefore, the pressure gradients and flow velocities at the vicinity of the well are approximated based on analytical or semianalytical formulas. Neglecting important near-well physics such as sharp pressure gradients, reservoir inhomogenity or completion geometry. To address this issue methods have been developed to advance the near-well representation by improving the standard Cartesian grid technique. In order to increase the accuracy of the numerical grid's resolution, a local grid refinement (LGR) method was proposed (Heinemann, et al., 1991) using irregular PEBI (perpendicular bisection method) grid. It was proved for vertical wells that the LGR method helps to capture the near-well flow patterns, while the PEBI unstructured grid significantly increases the flexibility of the spatial discretization. A similar unstructured LGR method was presented recently, with upscaling in the near-well area (Karimi-Fard \& Durlofsky, 2012). This expanded well modeling approach was designed to capture the key near-well

1 - Technical University of Denmark, DK-28oo Kgs. Lyngby, Denmark

2 - Lloyd's Register, DK-29oo Hellerup, Denmark

3 - Computational Science and Engineering Laboratory, ETH Zürich, CH-8og2 Zürich, Switzerland 
effects. The model itself is constructed through a single-phase steady-state solution on the underlying fine-scale model, in which certain key features such as hydraulic fractures can be resolved. A new global upscaling method was also introduced for computing coarse scale transmissibilities. The applicability of the expanded well model was shown for a horizontal well problem and found to be in a close agreement with the reference solutions.

These methods are proven to be useful to increase the accuracy at the near-well area; however they still ignore the well completion and the flow in the wellbore. Today's computational capacity enables the use of Computational Fluid Dynamics (CFD) simulations on wells with the drainage area resolved, in a single three-dimensional (3D) numerical model. In CFD the small details of the well and completion can be resolved using small grid sizes, and the formation damage or reservoir heterogeneity may also be captured around the well. This enables a far more detailed representation of both the well and the near-wellbore area, leading to a potentially better inflow performance estimation.

Recent papers of Byrne (2009) introduced the use of CFD for modeling well inflow to a perforated openhole vertical well. Byrne showed that CFD can resolve the formation damage of asymmetric distribution around the well (Byrne, et al., 2010), and can capture the cross-flow appearing among adjacent layers for a heterogeneous layered reservoir (Byrne, et al., 2011). Furthermore, a case study was published about the detailed modeling of a perforated horizontal well, offshore Australia (Byrne, et al., 2014). Moreover, Molina (2015) have used CFD for investigating the performance of a perforated gas well using a detailed near-wellbore model, focusing on different completion methods and erosive effects on the wellbore (Molina, 2015).

The present work considers a steady-state singlephase application of CFD for horizontal well modeling, using Ansys Fluent v17 software. Furthermore, it aims to present the applicability of CFD for simplified well inflow problems where the flow is resolved in both the near-well reservoir area and in the wellbore, using fine numerical grid with a smooth transition between the well and the far reservoir. No upscaling method is considered, since CFD can resolve the formation heterogeneity when data is available. In order to prove the applicability of CFD on well inflow simulations, certain benchmark cases are considered. Afterwards, a horizontal producer well is modeled having $1 \mathrm{~km}$ long perforated producing section, draining from the Siri field offshore Denmark. In order to address the uncertainties arising from potential formation damage, different scenarios are taken into account. For all the cases the $3 \mathrm{D}$ flow field around the well are of interest, as well as the inflow profiles and productivity index of the well.

\section{Methodology}

\subsection{Governing equations}

The equations governing the isothermal fluid motion are the fundamental principles of mass and momentum conservation. Additionally, equations accounting for the transport of turbulence properties were also used.

Mass conservation:

$$
\frac{\partial \rho}{\partial t}+\frac{\partial\left(\rho u_{i}\right)}{\partial x_{i}}=0
$$

where $\rho$ is the mass of the fluid for unit volume, $u_{i}$ is the fluid velocity in a given spatial direction, $x_{i}$ is a spatial direction.

Momentum conservation for incompressible steady-state, Newtonian fluid flow:

$$
\rho\left(u_{j} \frac{\partial u_{i}}{\partial x_{j}}\right)=-\frac{\partial p}{\partial x_{i}}+\mu \frac{\partial^{2} u_{i}}{\partial x_{j}^{2}}+\rho g_{i}+S_{i}
$$

where, $p$ is pressure, $\mu$ is dynamic viscosity of the fluid, $g_{i}$ is the gravitational acceleration in a spatial direction, $S_{i}$ is the momentum source/sink term.

Due to the turbulent flow regime present in the well, transport equations of the standard $k-\varepsilon$ turbulence model were used as well (Ferziger \& Peric, 2002).

\subsection{Porous media}

The present CFD simulations only resolves the macroscopic flow patterns of the porous media and neglects the pore-scale flow. Thus, control volumes were defined all over the domain (grid cells), and averaged quantities over these volumes were considered. Thereby, in the porous domain the superficial velocity was used for the equations, similarly to the Darcy law.

The porous media model in Ansys Fluent used the Navier-Stokes momentum equation with the DarcyForchheimer equation as a momentum sink on the right hand side of the momentum balance equation:

$$
S_{i}=-\left(\frac{\mu}{k_{i}} u_{i}+\beta \rho\left|u_{i}\right| u_{i}\right)
$$

where $k_{i}$ is the viscous resistance (permeability) in a spatial direction, $\beta$ is the inertial resistance.

The momentum sink represents the pressure drop across the porous media which is arising due to viscous forces (Darcy term) and/or inertial forces (Forchheimer term). The inertial Forchheimer term is 
quadratic in velocity, therefore it is expected to become significant only for high velocities, which will not likely to occur for liquid flows. Thus, this term was neglected for all simulations of this study.

\subsection{Turbulence treatment}

In Ansys Fluent the porous medium had no effect on the turbulence generation or dissipation rates. In addition, the generation of turbulence was set to zero in the porous domain. Therefore, any specified turbulence property at the inflow boundaries were transported through the porous medium without any change, and the flow here was treated as laminar flow.

Whereas, in the free-flow zone (well, perforations) the transport of turbulent quantities were considered by using the Standard k- $\varepsilon$ turbulence model with Standard Wall Functions at the vicinity of the walls. Thus, attention was taken to keep the dimensionless wall distance $(y+)$ between 30 and 300 , to fulfill the requirements.

\subsection{Solver}

Computational Fluid Dynamics is a numerical procedure to seek approximate solutions for fluid flow related problems. The core of this procedure is to discretize the continuous domain on which the flow variables can be defined and the differential equations can be approximated by a set of algebraic equations, which can be solved by a computer.

Ansys Fluent uses a control volume based technique to convert the governing equations to algebraic equations. This technique consists of integrating the governing equations about each control volume, to yield discrete equations that conserves each quantity on a control volume basis. The present CFD simulations use a pressure-based (segregated) solver. This method solves the governing equations sequentially and iteratively due to the nonlinear, coupled formulas until a convergent numerical solution is obtained. Each iterations consists of the steps shown in Figure 1.

For computing the cell-face pressures, the standard interpolation scheme was selected. While the secondorder upwind interpolation was used for the momentum. equations. The pressure-velocity coupling is achieved by using the SIMPLE algorithm (semi-implicit method for pressure linked equations)(Patankar, 1980). Such algorithm is necessary, because the velocities obtained from solving the momentum equations may not satisfy the continuity equation locally, thus a pressure correction is computed to update the pressure and mass fluxes achieving mass conservation.

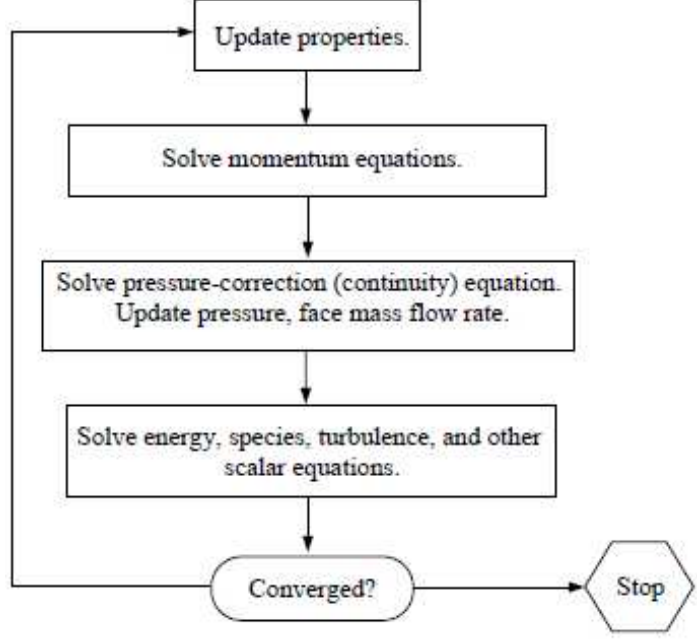

Figure 1: Flowchart of pressure based segregated solver

\section{Model parameters for the benchmark cases}

\subsection{Reservoir data}

The benchmark cases are based on the data of an open-hole horizontal well draining from the Troll field, described by Ozkan (1999). Two cases were considered, one assuming uniform well pressure from heel to toe (infinite well conductivity), and the other, considering the well with certain resistance to the flow inside (finite well conductivity). The data for the latter case is shown in Table 1.

Table 1: Troll field data, finite conductivity case

\begin{tabular}{llll}
\hline Formation thickness & $h$ & $\mathbf{2 2}$ & $\mathrm{m}$ \\
Drainage radius & $r_{e}$ & 850 & $\mathrm{~m}$ \\
Well length & $L$ & 800 & $\mathrm{~m}$ \\
Well radius & $r_{w}$ & 7.6 & $\mathrm{~cm}$ \\
Vertical position of well & $z_{w}$ & 3.5 & $\mathrm{~m}$ \\
Horizontal permeability & $k_{h}$ & 8500 & $\mathrm{mD}$ \\
Vertical permeability & $k_{v}$ & 1500 & $\mathrm{mD}$ \\
Oil density & $\rho_{o}$ & 881 & $\mathrm{~kg} / \mathrm{m}^{3}$ \\
Oil viscosity & $\mu_{o}$ & 1.43 & $\mathrm{cP}$ \\
Oil compressibility & $c_{o}$ & $1.0 \mathrm{e}-4$ & $1 / \mathrm{bar}$ \\
Total production & $q_{w}$ & 30,000 & $\mathrm{bbl} / \mathrm{d}$ \\
Reservoir pressure & $P_{e}$ & 158.6 & $\mathrm{bar}$ \\
\hline
\end{tabular}

The same properties were used for the infinite well conductivity case, except that the well was located centrally in the vertical plane of the reservoir $\left(z_{w}=11\right.$ $\mathrm{m})$, and uniform well pressure of 156.6 bar was applied on the sand-face as a boundary condition, obtaining a uniform drawdown of 2 bar. In addition, different well lengths were tested: 200, 400, 60o, 8oom. 

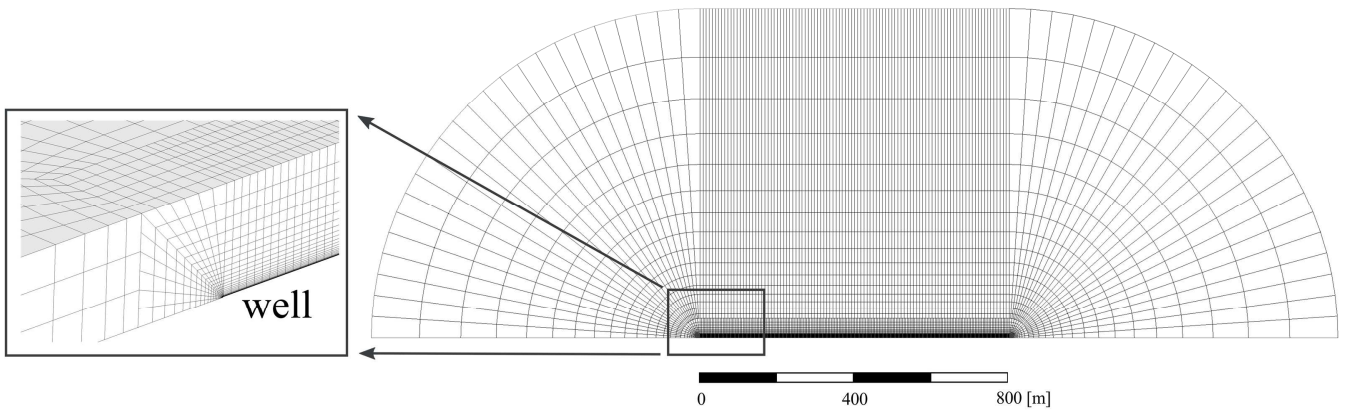

Figure 2: Spatial discretization of the benchmark reservoir for the infinite conductivity model. Overview of the multizone mesh. Right: plan view. Left: heel of the horizontal well is enlarged. Due to the symmetrical well placement only one quarter of the domain is modeled with symmetry boundary conditions on the sides

\subsection{Reservoir geometry}

The present single-phase steady-state simulations focused on the production from an oil saturated reservoir layer which is bounded by impermeable shale layers from top and bottom sides. Both boundary surfaces were modeled as flat impermeable walls parallel to each other and to the center-line of the well.

The shape of the drainage area for the CFD simulations at the horizontal plane were determined based on Joshi's argument (Joshi, 1990): "A horizontal well can be looked upon as a number of vertical wells drilled next to each other and completed in a limited payzone thickness. Therefore each end of a horizontal well would drain a circular area with a rectangular area at the center". Thus, the drainage radius given by Ozkan is enough to determine the location of the edge of the domain.

\subsection{Spatial discretization}

Due to the large difference in spatial scales a multizone hexahedral mesh was used, which is indicated on Figure 2. The multizone method requires to split up the domain to sweepable bodies that can be meshed individually. This way it is possible to use large elements in the reservoir far from the well, and smaller elements close to the well.

The finite conductivity model includes the horizontal well in the domain, thereby attention was taken to achieve conforming mesh topology among the well and the near-wellbore area in order to avoid stability issues during the simulation.

\subsection{Boundary conditions}

The symmetrical well placement enables the reduction of the reservoir domain. Thus, the domain for the infinite and finite conductivity models were quartered and halved respectively. Symmetry boundary condition was applied on the cutting planes.
It was assumed that impermeable layers are bounding the reservoir, therefore at the top plane of the domain no-slip wall boundary condition was applied (due to the symmetry the same applies for the bottom of the reservoir as well).

Constant pressure inlet was applied at the edge of the reservoir at $r_{e}=850 \mathrm{~m}$. The outlet was set as constant production rate at the heel of the well for the finite conductivity case. Whereas, constant pressure was set at the well sand-face for the infinite conductivity case.

\section{Results for benchmark case}

\subsection{Benchmark cases - Infinite conductivity well}

Horizontal wells are often modeled as infinitely conductive wells having uniform pressure from heel to toe. This assumption is justified on the basis that the pressure drop in the wellbore is negligible compared to the drawdown in the reservoir. Using such assumption it is enough to model the flow in the reservoir and neglect the well hydraulics.

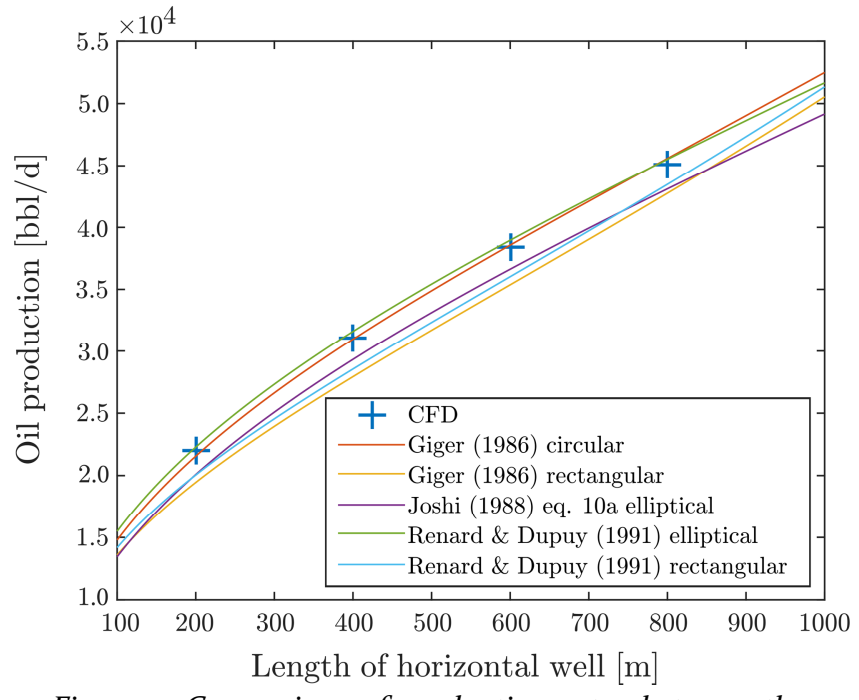

Figure 3: Comparison of production rates between the CFD numerical and five analytical models, for infinite conductivity horizontal wells (Troll field). Steady-state 
single phase problem for different well lengths. Drainage area increased proportionally with the well lengths.

Five single-phase steady-state analytical models were used for benchmarking. Giger et al. (1984) reported two formulas for estimating well inflow for circular and elliptical drainage areas. Joshi (1988) proposed a similar formula assuming elliptical drainage area. Renard and Dupuy (1991) reported two equations assuming elliptical and rectangular drainage areas. Using these formulas the oil production was estimated on the Troll field for varying well lengths between 100 and $1000 \mathrm{~m}$, assuming central well placement in both the horizontal and vertical planes.

Figure 3 summarize the production rates of the horizontal well, computed with numerical (CFD) and analytical methods. The figure clearly shows, that the numerical results are in a good agreement with the analytical solutions. The deviation from the analytical results is $0.1 \%$. Note, that the drainage area was increased proportionally with the well length, and was adjusted to reach the same magnitude for all the analytical and numerical methods.

\subsection{Benchmark cases - Finite conductivity well}

The drawdown in the reservoir for long horizontal wells may become the same order of magnitude as the pressure difference along the well. In such cases, the infinite conductivity assumption fails, and the well hydraulics must be included in the model.

A semi-analytical solution was proposed by Ozkan (1999) on the Troll field. These results were used as the basis of comparison for the CFD numerical model.

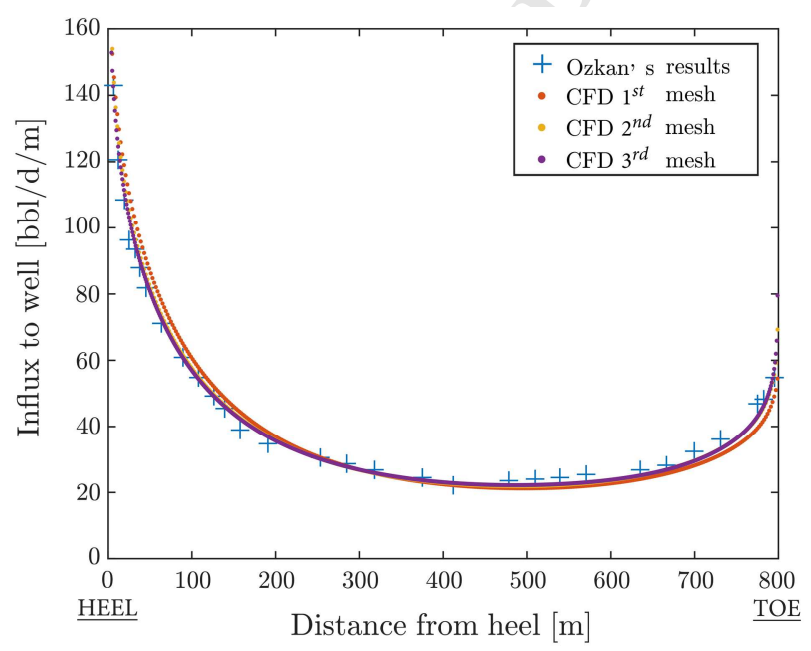

Figure 4: Inflow profiles along the well length. Comparison of CFD results (for different numerical grids) with Ozkan's semi-analytical results(Ozkan, et al., 1999). Steady-state single-phase simulation of a well draining from the Troll field.
The results are shown in Figure 4 by indicating the inflow profile along the well length for the semianalytical and for three numerical solutions with different grids. One may note that the inflow profile of the CFD simulation is within $\mathbf{1 - 2} \%$ of the results of Ozkan (1999). The numerical mesh 1, 2 and 3 have cell counts as follows: 70,000; 300,000; 700,000 cells. The refinement of the mesh was the strongest at the well and near-wellbore area in the radial direction (from well's axis), where the gradients are the highest thus, where the accurate resolution was most important.

It can be concluded that the numerical investigations with CFD can meet the results of the well-known analytical formulas with great accuracy. Thus, CFD may be used for modeling complex well inflow problems.

\section{Model parameters for the Siri field}

\subsection{Reservoir data}

The Siri field is located in the North-Western part of the Danish sector of the North Sea. It lies at a depth of $2100 \mathrm{~m}$ in Palaeocene sandstone. The production started in 1999. The most important reservoir parameters can be found in Table 2.

Table 2: Reservoir parameters of Siri field

\begin{tabular}{llll}
\hline Formation thickness & $h$ & 27 & $\mathrm{~m}$ \\
Drainage radius & $r_{e}$ & 600 & $\mathrm{~m}$ \\
$\begin{array}{l}\text { Drainage area } \\
\text { Mean of isotropic }\end{array}$ & $A_{d}$ & 2.33 & $\mathrm{~km}^{2}$ \\
permeability & $\bar{k}$ & 178 & $\mathrm{mD}$ \\
$\begin{array}{l}\text { Standard dev. of isotropic } \\
\text { permeability }\end{array}$ & $\sigma_{k}$ & 78 & $\mathrm{mD}$ \\
Reservoir pressure & & & \\
Vertical well location & $p_{e}$ & 230 & $\mathrm{bar}$ \\
Producing well length & $h_{w}$ & 4 & $\mathrm{~m}$ \\
Oil density & $L_{w}$ & 1000 & $\mathrm{~m}$ \\
Oil viscosity & $\rho_{o}$ & 844 & $\mathrm{~kg} / \mathrm{m}^{3}$ \\
Oil compressibility & $\mu_{o}$ & 1.0 & $\mathrm{cP}$ \\
Oil saturation & $c_{o}$ & $1.8 \mathrm{e}-4$ & $1 / \mathrm{bar}$ \\
\hline & $S_{o}$ & 1.0 & - \\
\hline
\end{tabular}

Information about the reservoir permeability was available in the ECLIPSE reservoir simulation model of the Siri field, provided by DONG Energy. The isotropic permeability values were taken from the well cells of the simulator. A ${ }_{1} \mathrm{D}$ polynomial regression was done to fit a continuous function on the discrete points obtained from ECLIPSE. This function describes the variation of isotropic permeability along the axial distance, parallel to the well. Such a simplified method represents the formation heterogeneity in the near-wellbore area (o$100 \mathrm{~m}$; see Figure 12), which is essential to properly 
estimate the inflow to the well. Whereas, far from the well (100-60o $\mathrm{m}$ ) the mean isotropic permeability was used $(\bar{k}=178 \mathrm{mD})$, and the formation was modeled as homogeneous.

The drilling induced formation damage was taken into account by modeling the filtrate invaded zone as an impaired permeability zone around the well with $k_{\text {skin }}=0.1 k_{\text {res }}$. The presence of any mud cake was omitted since its effect on the production of a perforated well is negligible.

Three formation damage scenarios were taken into account: no damage, shallow filtrate invasion and medium filtrate invasion, as indicated in Table 3. Note, that the penetration of the perforations to the formation was presumed to be $20 \mathrm{~cm}$, thus the medium invasion extends beyond the tip of the perforations.

Table 3: Formation damage scenarios

\begin{tabular}{lll}
\hline & \multicolumn{2}{l}{ Penetration } \\
$\mathbf{k}_{\text {skin }} / \mathbf{k}_{\text {res }}$ \\
\hline No damage & - & 1.0 \\
Shallow invasion & $12 \mathrm{~cm}$ & 0.1 \\
Medium invasion & $40 \mathrm{~cm}$ & 0.1 \\
\hline
\end{tabular}

\subsection{Reservoir geometry}

The single-phase steady-state simulations of the Siri field focused on the production from an oil saturated reservoir layer which is bounded by impermeable shale layer from the top and oil-water contact from the bottom. However, both surfaces were modeled as flat impermeable walls parallel to each other and to the center-line of the well, during the production.

The short thickness of the reservoir is clearly drained by the well entirely. However, defining the lateral extent of the drainage area is more challenging. The edge of the reservoir was set as constant pressure boundary due to the steady-state flow regime. However, this boundary cannot meet the real pressure contour which would develop in the reservoir during production. Therefore, the inflow boundary must be placed "far enough" from the well, thus it may not impact the pressure contours developing in the reservoir during production. This "far enough" distance was investigated here with a series of CFD simulations. As the drainage radius was increased systematically, and the well inflow profile was monitored.

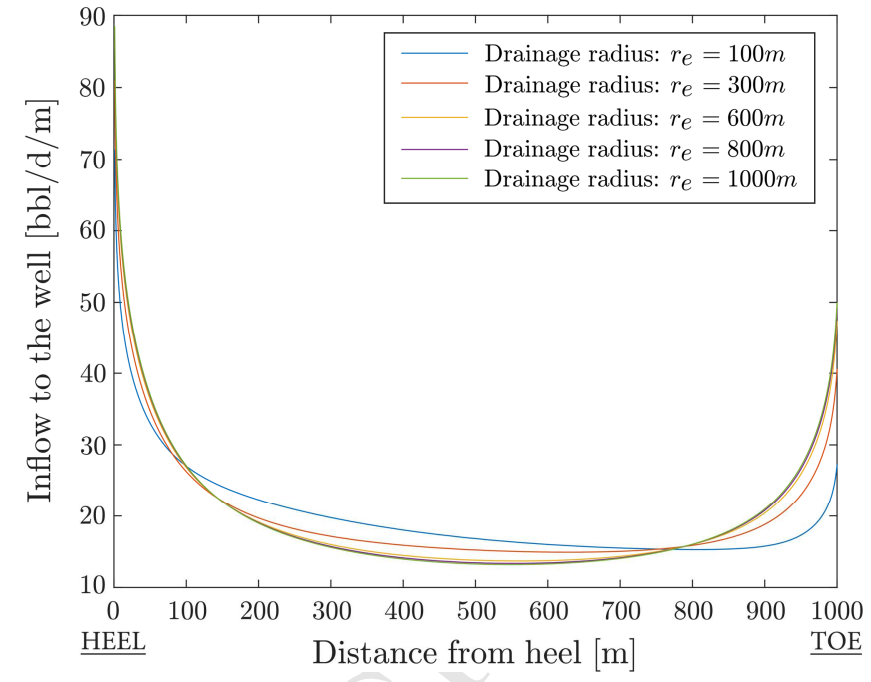

Figure 5: Inflow profiles for various drainage radiuses. Constant inflow pressure of 230 bar, constant production rate of $20,000 \mathrm{bbl} / \mathrm{d}$. Homogeneous reservoir assumed.

The simulations were undertaken with high production rate of $20,000 \mathrm{bbl} / \mathrm{d}$ at the heel, and fixed reservoir pressure. Furthermore, the entire reservoir was modeled to be homogeneous, using the mean isotropic permeability of $178 \mathrm{mD}$. The resulting inflow profiles can be seen on Figure 5 .

The distribution of the inflow differs considerably for the smaller drainage areas, however they are converging upon reaching the drainage radius of 600 $\mathrm{m}$. Therefore, this value was selected as the drainage radius of the horizontal well, and it is concluded that the inflow pressure boundary lies far enough, and thus it will not impose an erroneous inflow profile on the well.

\subsection{Well completion geometry}

The numerical simulations only include the producing section of the horizontal well, modeled with a straight centerline, located $h_{w}=4 \mathrm{~m}$ from the top reservoir boundary. The well is cemented and perforated along the $1 \mathrm{~km}$ long producing section with the parameters given in Table 4 .

\section{Table 4: Well completion parameters}

\begin{tabular}{llll} 
Wellbore diameter & $d_{w b}$ & 21.6 & $\mathrm{~cm}$ \\
Production liner diameter & $d_{w}$ & 14.0 & $\mathrm{~cm}$ \\
Well length & $L_{w}$ & 1000 & $\mathrm{~m}$ \\
Perforation density & $s_{d}$ & 4 & $\mathrm{spf}$ \\
Perforation phasing & $\phi_{p}$ & 180 & 0 \\
Perforation diameter & $d_{p}$ & 2 & $\mathrm{~cm}$ \\
Perforation penetration & $l_{p}$ & 20 & $\mathrm{~cm}$ \\
Prod. liner surface roughness $\epsilon$ & 1 & $\mathrm{~mm}$ \\
\hline
\end{tabular}

The numerical resolution of the well completion has a considerable impact on the computational cost when simulating long wells. Therefore, a cost 
efficient way of modeling the completion was sought by considering different numerical resolutions on short well sections. It was found that it would have been too expensive to resolve all $\sim 13,000$ perforations individually, therefore a reduced order numerical model was proposed that models the presence of the perforations with a continuous high permeability "porous perforation channel", as it can be seen on Figure 6. This channel extends from heel to toe in the horizontal direction along the well and had thickness and penetration equal to the real perforations.

The permeability of the porous perforation channel was set to meet the same overall productivity as the detailed model (with the resolved perforations) would have, with the given completion properties. Additionally, the surface roughness of the well was tuned to compensate the presence of the perforation cavities in the production liner. For this reason short well sections $(50 \mathrm{~m})$ were simulated to tune both the porous perforations permeability and the well surface roughness for all three of the formation damage scenarios.
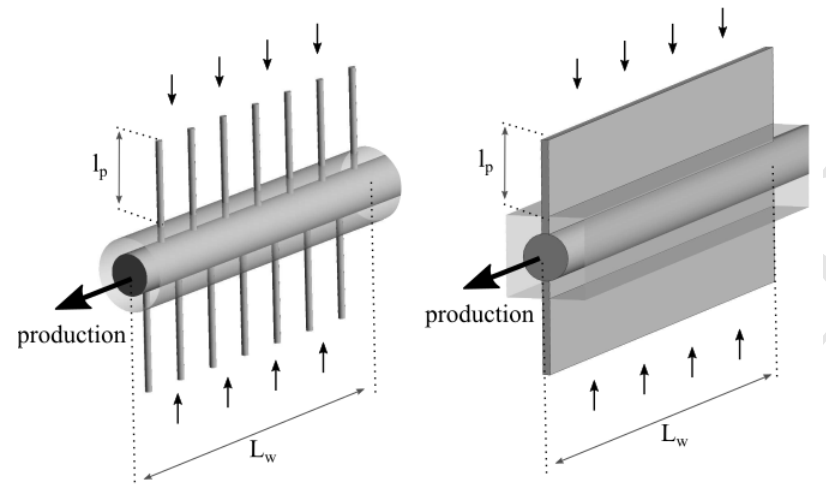

Figure 6: Horizontal well geometries. Left: detailed model with resolved perforations. Right: reduced model with modeled perforations (porous perforations channel). Note, that for both models, the fluids can enter the well across the entire surface of the perforations.

Figure 7 indicates the pressure field around the short well sections for comparison. One may see that the pressure contours are comparable, however for the reduced order model the contours remain radially symmetrical close to the well due to the lack of the individual perforations.
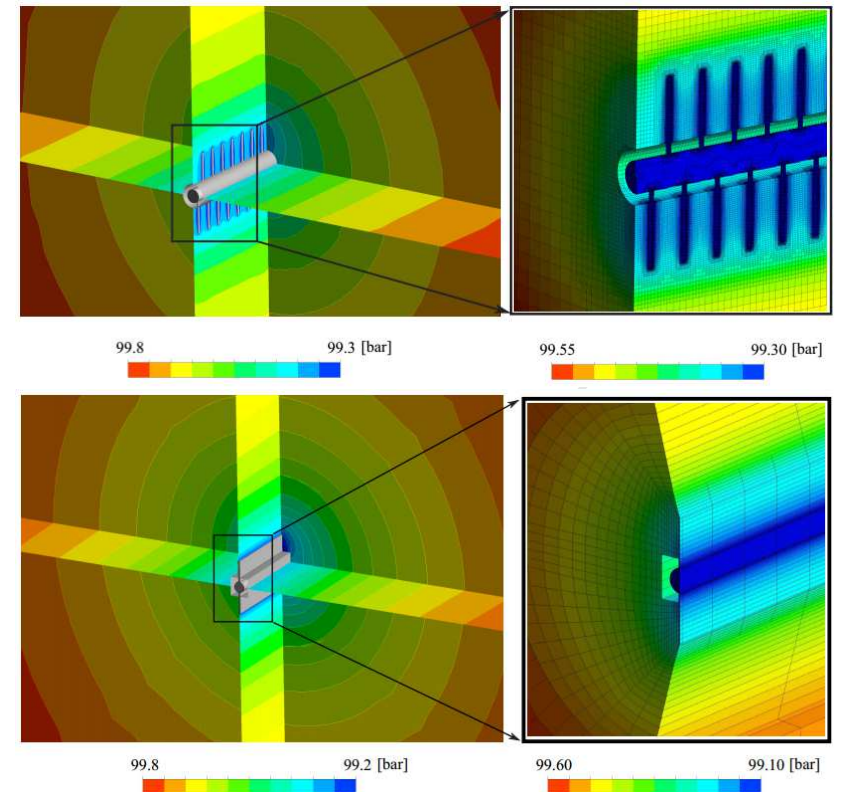

Figure 7: Pressure field around short well sections. Top: perforations resolved (detailed model). Bottom: porous perforation channel (reduced order model). Right side of the figures indicates the pressures in the vertical cross section intersecting the well.

As a result of the simulations with the short well sections, the permeability of the perforation channel is indicated in Table 5 as a fraction of the reservoir permeability for all three formation damage scenarios. The reservoir and the formation damage permeability varies with the well axial distance, thus the permeability of the porous perforation channel has to be varied as well, to keep the indicated ratio fixed. This way the permeability in two directions perpendicular to the well axis was determined. While the permeability parallel to the well axial direction was set to zero, to prevent any flow in the channel parallel to the well stream.

Table 5: Porous perforation channel permeability as a fraction of the reservoir permeability. Using these fractions the reduced numerical model can meet the productivity of the detailed model

\begin{tabular}{ll}
\hline Formation damage scenarios & $\mathbf{k}_{\text {perf }} / \mathbf{k}_{\text {res }}$ \\
\hline No damage & 32.58 \\
Shallow invasion & 23.59 \\
Medium invasion & 3.26 \\
\hline
\end{tabular}

The obtained ratios were found to be correct for different flow rates and reservoir permeabilities as well, as it is thought to be influenced by the well completion only. Therefore, it can be concluded that such a reduced order model of the perforated well completion can potentially be used to represent long horizontal wells having $180^{\circ}$ phasing perforated completion. 


\subsection{Spatial discretization}

The current study includes both the well and its drainage area in one numerical model, which makes the grid generation challenging due to the large differences in spatial scales. The dimensions in the porous drainage volume are in the order of $10^{3} \mathrm{~m}$ in the horizontal and $10^{1} \mathrm{~m}$ in the vertical plane, whereas the well diameter is in the order of $10^{-1} \mathrm{~m}$. Therefore, to resolve both scales with minimized cell count, the grid cell sizes had to be increased incrementally from the well - where the small details are of interest - towards the far reservoir. Furthermore, due to its larger volume and potentially better aspect ratio, hexahedral cells were used instead of tetrahedral cells. Hexahedral cells result in high quality mesh and high accuracy (Ferziger \& Peric, 2002).

The numerical grid was built up of hexagonal elements of various sizes, using the multizone hexahedral meshing method. The domain was decomposed until sweepable bodies were obtained (indicated with different colours on Figure 8) on which the structured hexahedral mesh can be generated. Among the bodies the mesh topology is conforming, however when sharper transition of mesh size is sought interface elements needs to be used to create non-conforming mesh topology. Note, that the solution may become unstable when the mesh topology is non-conforming at the porous non-porous interface, thus it is essential that the well and its close vicinity are meshed in one part.

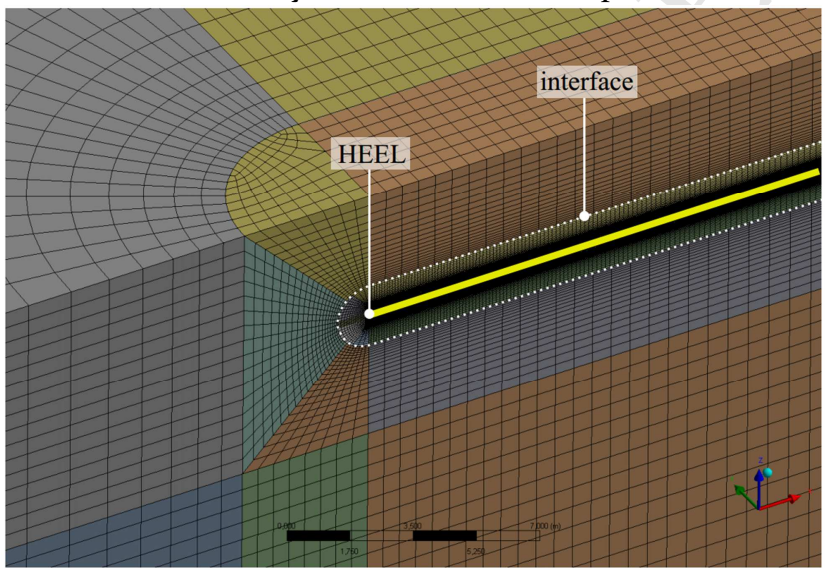

Figure 8: Spatial discretization of the simulation domain. Half of the drainage volume is shown in the vicinity of the heel of the well. The well is indicated by yellow, the interface between the two mesh densities are shown with dashed white line.

The near-well area needs a fine resolution due to the steep pressure and velocity gradients. Thus, here small cell sizes were used with cylindrical symmetry, surrounding the horizontal well (Figure 9). The mesh in the production liner was conforming to the mesh of the perforation and built up of 360,000 cells. Close to the wall, inflation layers were used to capture the steep velocity gradients arising from the rough noslip walls (Figure 9). The annular gap between the production liner outer wall and the wall of the wellbore is filled with cement in real world. Thereby, this area was not resolved by the numerical model, shown blank on Figure 9.

Further from the well the cell sizes increased, as the pressure gradient decreased and a lower resolution was also adequate in the reservoir.

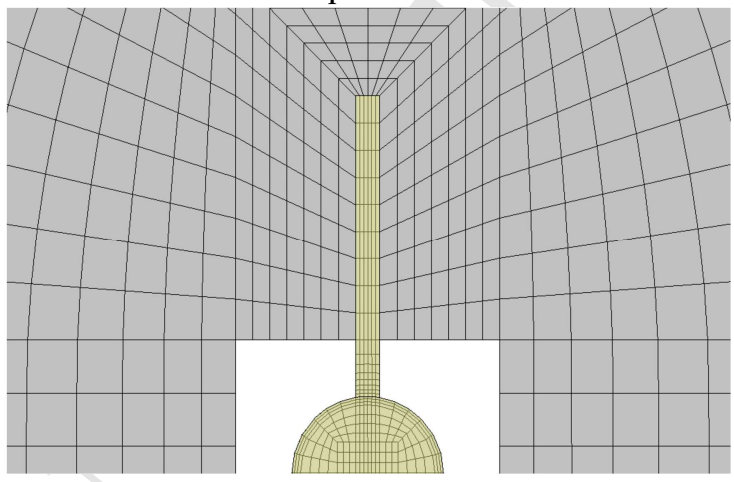

Figure 9: Mesh shown at the cross section of the horizontal well, perpendicular to the well axis. Yellow colour indicates the well and the porous perforation channel.

A series of simulations were undertaken to reveal the directional sensitivity of grid spacing on the solution. It was found that the mesh is most sensitive to the cell size in the radial direction, whereas the well axial direction requires a considerably lower resolution.

It must be noted that a considerable speedup can be achieved with using the reduced order well model, compared to the detailed model. While the detailed numerical model uses minimum 300,00o cells, the reduced order model uses only 4,ooo cells for im well section. Since here there is no need to capture the flow near and in the perforations. Such difference can be observed at the computational time as well. While the detailed model needs 10 hours to reach a converged solution, the reduced order model reaches solution within minutes. Furthermore, since the reduced order model has a uniform cross-section due to the continuous porous perforation, the cell sizes can be increased at the middle part of the well, where the flow patterns are less complex compared to the ends of the well. 


\subsection{Boundary conditions}

The boundary conditions are indicated on Figure 10. At the edge of the domain the inflow pressure was fixed to be 230 bar. The top and bottom boundaries of the formation were walls, whereas there was a symmetry boundary at the vertical plane intersecting the horizontal well, due to the symmetrical geometry and reservoir conditions.

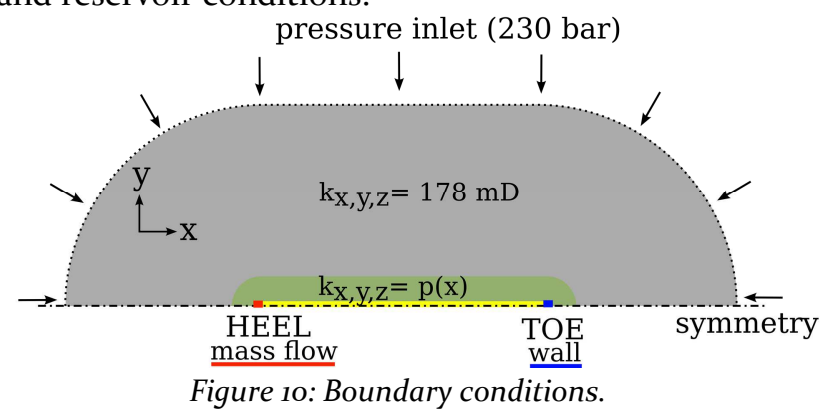

At the heel of the well the overall production rate was fixed by using a mass-flow boundary condition. The toe of the well was modeled as a wall. Three production scenarios were considered: 5,000; 10,000; 20,00o bbl/d.

The surface of the borehole was modeled as no-slip wall, whereas the surface of the production liner was a rough wall with $1.5 \mathrm{~mm}$ surface roughness and 0.7 roughness constant to account the considerable increase of roughness from corrosion or scale buildup during decades of production.

The well and the perforation channel were connected by the use of a porous jump face. This boundary condition ensures that the fluids can pass through the face but also keeps a no-slip condition from the well side. The connection of the perforation channel and the reservoir did not require any boundary condition, since both domains defined as porous regions.

The formation heterogeneity was taken into account in the o-100 $\mathrm{m}$ region from the well only. A polynomial function was used to describe the continuous variation of permeability (Figure 12). Whereas, further from the well the overall average permeability of $178 \mathrm{mD}$ was used to model the reservoir as a homogeneous domain (Figure 10).

After all, nine single phase steady-state simulations were conducted to model the performance of the horizontal well. Three formation damage scenarios were considered, each of which with three production rates. The most likely scenario was presumed to be the one with the shallow filtrate invasion producing with a rate of $10,000 \mathrm{bbl} / \mathrm{d}$.

\section{Results for Siri field}

\subsection{Flow field}

This section consider the results of the flow field in the near-well area by presenting pressure and velocity fields. In addition, the distribution of well inflow and productivity index are shown along the well length for different formation damage scenarios.

The drainage mechanism of the horizontal well is shown by pressure and velocity fields in Figure 11 and Figure 12 respectively. One can see that the pressure contours are elliptical far from the well (Figure 11), but deviate in the heterogeneous zone, which may also be observed at the streamline plot. Figure 12 indicates a similar deviation of the velocity magnitude scalar field. There are three locations with increased flow velocity: heel, toe, and a zone between 200-400 $\mathrm{m}$. The high velocity at the heel and toe results from the end effects of the well and can be explained by the partial penetration of the well into the domain. However, the increased flow velocity between 200-400 $\mathrm{m}$ and suppressed flow at $100 \mathrm{~m}$ indicates a direct correlation between the local permeability and the flow velocity in the near-well area as expected. 


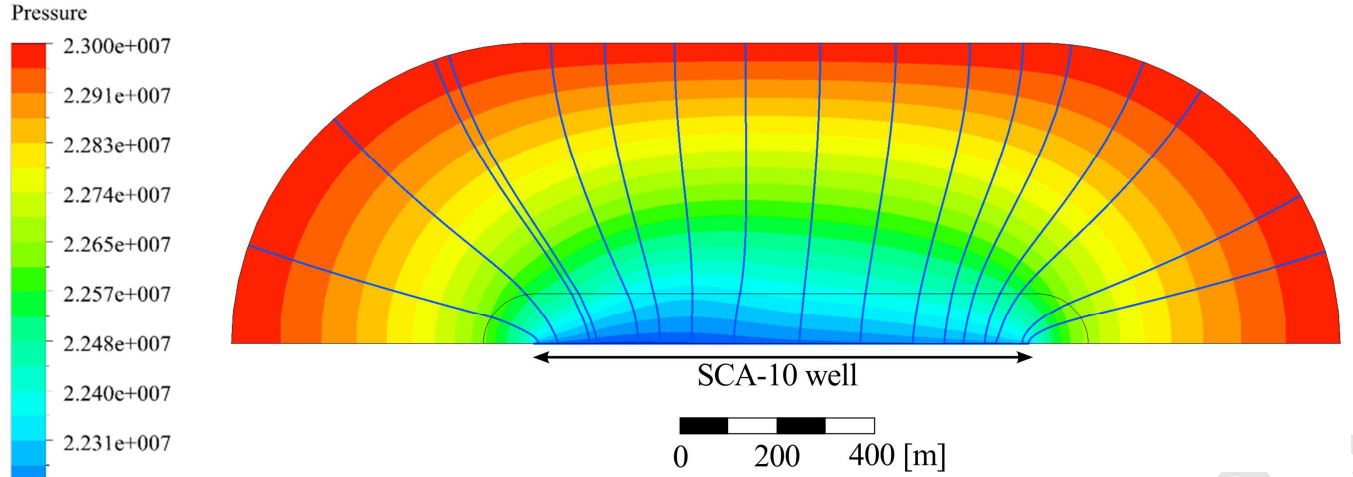

Figure 11: Pressure field and streamlines, shown at the horizontal plane. Black line indicates the $100 \mathrm{~m}$ zone from the well where the formation heterogeneity was captured. Shallow filtrate damage scenario, 10,00o bbl/d production rate. Siri field.
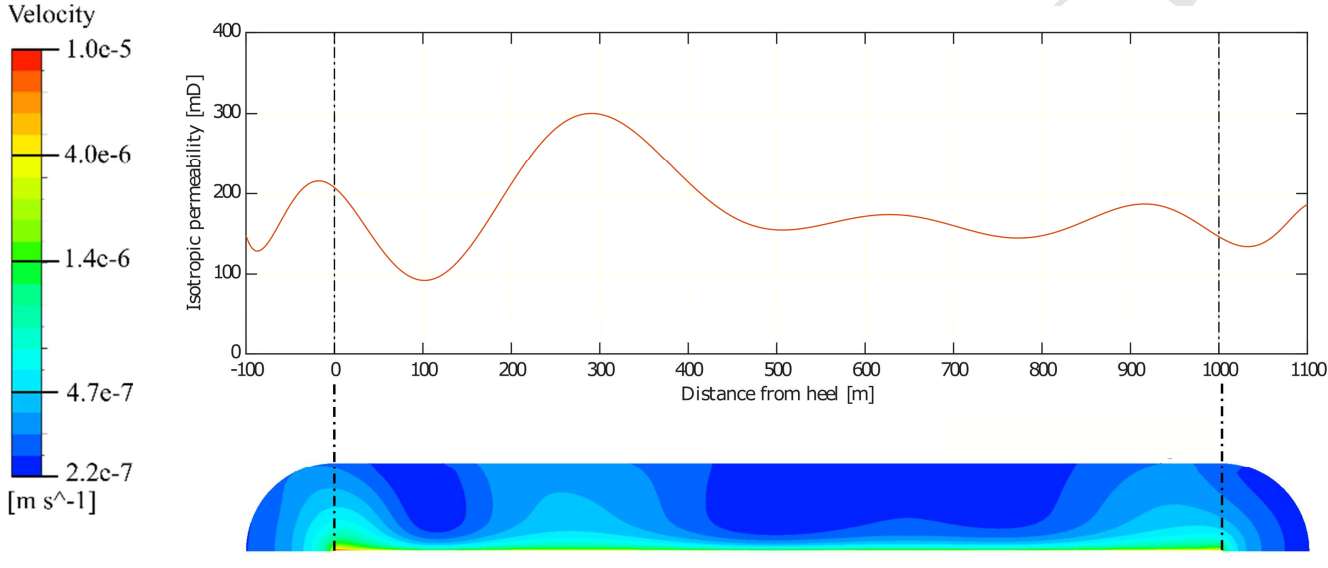

HEEL

$\underline{\mathrm{TOE}}$

Figure 12: Velocity magnitude indicated at the horizontal plane o-10o $\mathrm{m}$ zone from the well, together with the function describing the variation of permeability. High inflow velocity at the heel, toe and in the high permeability zone. Siri field.

\subsection{Inflow to the well}

The inflow to the well is presented here for every 1 $\mathrm{m}$ well section, to reveal its distribution along the well length for the three formation damage scenarios. Note, that the numerical simulations were undertaken with fixed production rate boundary condition at the heel of the well. Thus, the overall production rate is not affected by the presence of the formation damage.

Figure 13 shows the inflow to the well when the total production rate is $10,000 \mathrm{bbl} / \mathrm{d}$. One may see an uneven inflow distribution for all three scenarios, which is caused by the formation heterogeneity. Note that for a homogeneous reservoir the middle section of the inflow profile should be evenly distributed, as it can be seen in Figure 5. Furthermore, the increased inflow between 200-400 $\mathrm{m}$ in Figure 13 indicates that the production from the high permeability zones are considerably higher than from the adjacent zones. This is in a good agreement with the results shown in Figure 12.

There is no significant difference in the well inflow between the no-damage and the shallow filtrate invasion cases. However, the medium filtrate invasion cause the inflow profile to change considerably. This may also be seen in Figure 14, where the impact of the formation damage on the inflow profile is shown relative to the no-damage base case. The figure indicates a severe $-40 \%$ inflow reduction at the ends of the well, and a $+20 \%$ increased inflow at the middle of the well. Implying that the resistance of the filtrate invasion redistributes the flow around the well to feed the middle of the well stronger. Such flow redistribution seemed to be increasing with the extent of the formation damage. 


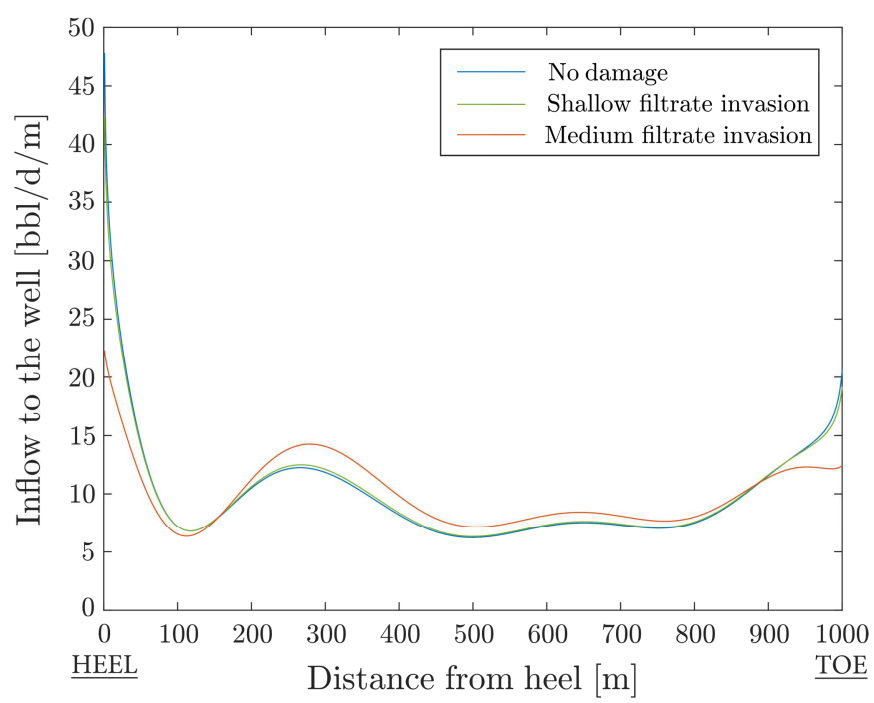

Figure 13: Inflow profile of the horizontal well, total production rate: 10,000 bbl/d. Three formation damage scenarios indicated. Siri field.

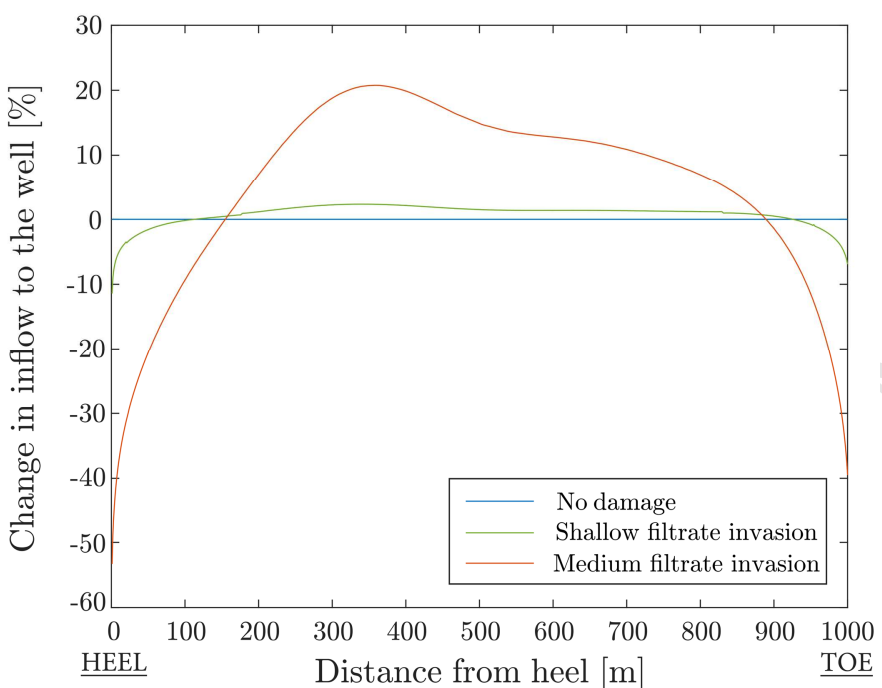

Figure 14: Change of the inflow profile of the well, total production rate: 10,00o $\mathrm{bbl} / \mathrm{d}$. The effect of formation damage relative to the no-damage base case. Siri field.

\subsection{Inflow performance}

The inflow performance of the well is addressed through its productivity index shown for $1 \mathrm{~m}$ well sections. It is computed by taking the ratio of the inflow $[\mathrm{bbl} / \mathrm{d} / \mathrm{m}]$ and the drawdown [bar] per $1 \mathrm{~m}$ well length.

The distribution of the productivity index is shown for $10,000 \mathrm{bbl} / \mathrm{d}$ total production rate in Figure 15, for all three formation damage scenarios. It can be seen that the most productive well segments are located at the ends and between 200-400 $\mathrm{m}$ in the middle of the well, where the reservoir permeability is high (see Figure 12). But in general, a similarity between the distribution of the curves of Figure 14 and Figure 16 may be observed, implying that the shape of the

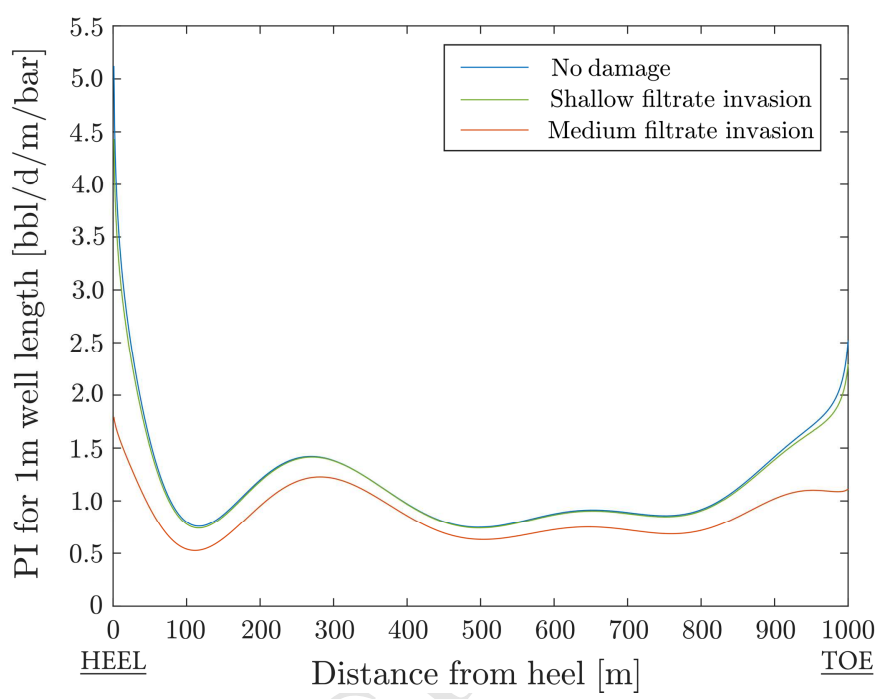

Figure 15: Distribution of the productivity index (for Im well sections) along the well length. Three formation damage scenarios indicated. Siri field.

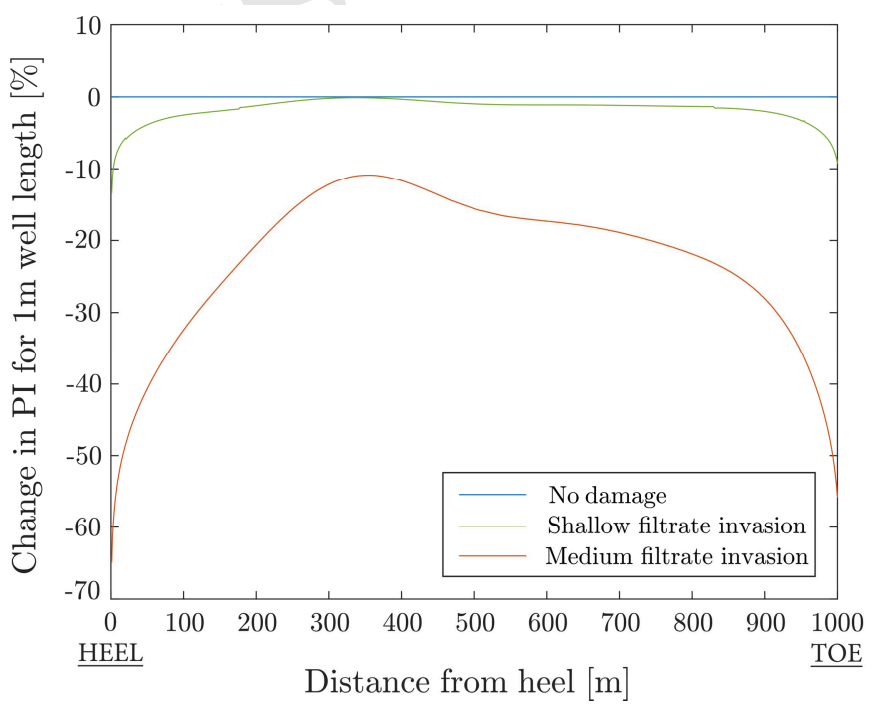

Figure 16: Change of the productivity index of the well. The effect of formation damage relative to the no-damage base case. Siri field.

productivity curve is mostly influenced by the inflow profile.

One may see the overall trend that any filtrate invasion decreases the productivity index at every point along the well (Figure 16). Furthermore, such a decrease of productivity is considerably stronger at the ends of the well than at the middle. Therefore, the presence of the formation damage evens out the well segment productivity, as it can be seen on Figure 15.

When considering the impact of the two filtrate invasion scenarios, it can be seen that the shallow invasion leaves the well productivity almost unaffected, similarly to the inflow profiles. Whereas, the medium filtrate invasion causes a severe reduction of $-50 \%$ at the ends and $-20 \%$ at the middle 
of the well. Note that the overall well productivity (fraction of the total production rate and drawdown at the heel of the well) decreased by $-2 \%$ and $-25 \%$ compared to the no damage case, for the shallow and medium filtrate invasion cases respectively.

\section{Discussion and Conclusion}

The present study has demonstrated that CFD is applicable for basic well inflow problems. It was proven that CFD can meet the results of the wellknown analytical formulas with an acceptable accuracy.

In addition, simulations with CFD enables the representation of the three dimensional flow field in the near-wellbore area, thus the resulting pressure and velocity fields can be used to interpret the well inflow-performance. As expected, the reservoir heterogeneity in the near-well area impacts the inflow to the well significantly. A correlation was observed between the reservoir permeability and the well segment productivity.

The presence of formation damage around the borehole was found to impact the well productivity stronger at the ends than at the middle of the well. The numerical simulations revealed that the redistribution of flow patterns was the underlying cause behind that phenomenon. This might be explained by considering the streamlines in the horizontal plane feeding the well. When the fluids are flowing through the formation damage region, they suffer a pressure loss proportional with the flow velocity - according to Darcy's law. Thus, the streamlines are redistributed to feed the middle of the well (where the inflow velocity is smaller) rather than the ends of the well (where the inflow velocity would be higher), to suffer a smaller pressure loss. Nonetheless, a considerably higher drawdown was necessary to drain the desired amount of fluid due to the presence of the filtrate damage, this decreased the well productivity at every point along the well, meaning that despite of the flow redistribution, the well segment productivity decreased everywhere along the well. Even if such a change of well productivity is uneven and higher toward the ends.

Furthermore, the results suggested that there is a distinct difference between the shallow and medium filtrate invasion scenarios. This is explained by considering the penetration of the perforations into the formation. Until the filtrate invasion is shallower than the perforations, the well productivity is affected less than $10 \%$. However, as the filtrate extends beyond the tip of the perforations, one may see a severe reduction of well productivity between 20$60 \%$.

The present study has focused on the single phase steady-state simulation of oil wells. However in real life the inflow to the well is more complicated, having multiple phases present. Such phenomenon may only be captured using unsteady simulations since the change in the reservoir saturation and preferential flow of certain phases can only be resolved with time dependent investigations. With multiphase CFD simulations, one may resolve the water cresting effect as the preferential flow of water breaks through at the heel of the well due to the locally high drawdown. Such simulations are challenging to carry-out since the relevant time steps are considerably different for the well (flow velocity: $10^{-1}-10^{\circ} \mathrm{m} / \mathrm{s}$ ) and for the reservoir (flow velocity: $10^{-7}-10^{-5} \mathrm{~m} / \mathrm{s}$ ), thus for engineering use one might want to consider coupling CFD with nodal simulation models to achieve robust numerical models.

\section{Acknowledgement}

The authors wish to thank the members of the OPTION (Optimizing Oil Production by Novel Technology Integration) project for their support. OPTION is a joint industry project between Lloyd's Register, DONG Energy, Welltec, InnovationsFonden - Denmark, Technical University of Denmark, and the University of Copenhagen.

\section{References}

Byrne, M., Djayapertapa, L., Watson, K. \& Goodin, B., 2014. Complex Completion Design and Inflow Prediction Enabled by Detailed Numerical Well Modeling. Lafayette, SPE 168149 .

Byrne, M., Jimenez, M. A. \& Chavez, J. C., 2009. Predict Well Inflow Using Computational Fluid Dynamics - Closer to the Truth?. Scheveningen, SPE 122351.

Byrne, M., Jimenez, M. A., Rojas, E. A. \& Chavez, J. C., 2010. Modeling Well Inflow Potential in Three Dimensions Using Computational Fluid Dynamics. Lafayette, SPE 128082.

Byrne, M., Jimenez, M. A., Rojas, E. \& Castillo, E., 2011. Computational Fluid Dynamics for Reservoir and Well Fluid Flow Performance Modeling. Noordwijk, SPE 144130. 
Ferziger, J. H. \& Peric, M., 2002.

Computational Methods for Fluid Dynamics. 3

ed. s.l.:Springer.

Giger, F., Reiss, L. \& Jordan, A., 1984. The reservoir engineering aspects of horizontal drilling. Houston, SPE 13024.

Heinemann, Z. E., Brand, C. W., Munka, M. \& Chen, Y. M., 1991. Modeling Reservoir Geometry With Irregular Grids. SPE Reservoir Engineering, May, pp. 225-232.

Joshi, S., 1990. Horizontal Well Technology. Tulsa: PennWell Books.

Joshi, S. D., 1988. Augmentation of Well Productivity With Slant and Horizontal Wells. Journal of Petroleum Technology, pp. 729-739.

Karimi-Fard, M. \& Durlofsky, L. J., 2012. Accurate Resolution of Near-Well Effects in Upscaled Models Using Flow-Based Unstructured Local Grid Refinement. SPE Journal, December, p. 1084.

Molina, O. M., 2015. Application of Computational Fluid Dynamics to NearWellbore Modeling of a Gas Well. s.l.:s.n.

Ozkan, E., Sarica, C. \& Haci, M., 1999. Influence of Pressure Drop ALong the Wellbore on Horizontal-Well Productivity. SPE Journal, 4(3), pp. 288-301.

Patankar, S., 1980. Numerical heat transfer and fluid flow. s.l.:Hemisphere Publishing Corporation.

Renard, G. \& Dupuy, J., 1991. Formation Damage Effects on Horizontal-Well Flow Efficiency. Journal of Petroleum Technology, pp. 786-789. 